\title{
COMPARISON THEOREMS FOR GRAVITY WAVES IN BASINS OF VARIABLE DEPTH*
}

\author{
BY \\ CHIA-SHUN YIH \\ University of Michigan
}

\begin{abstract}
Surface waves of a homogeneous liquid and internal waves of a stratified liquid in basins of variable depth are considered. Inequalities involving the frequencies of oscillation are obtained when a container with one size or geometry is compared with another with a different size or geometry, when waves with one wavelength are compared with waves with another wavelength, or when one stratification is compared with another. Since exact solutions for gravity waves in basins of variable depth are so rare, one hopes the comparison theorems presented herein will be useful.
\end{abstract}

1. Introduction. The number of explicit analytical solutions for water waves in channels of variable depth is extremely small. The few solutions in Lamb's book [1] (pp. 442-450) remain to this day the only ones in existence. The situation with internal waves is quite similar; the only explicit analytical solutions extant are for linearly or exponentially stratified fluid in ellipsoids and elliptic channels, when the Boussinesq approximation is used. For this reason it is desirable to have some comparison theorems that enable one to estimate the frequency of oscillation reliably and quickly, for all modes of wave motion.

In this paper a few such theorems will be given, when one size or geometry is compared with another, one wavelength with another, or one stratification with another. Some of the theorems are fairly obvious, but in combination with the less obvious ones give a powerful means of locating the range in which the frequency must lie.

2. System governing surface waves. Consider first surface waves in a homogeneous liquid contained in a basin of arbitrary shape. The fluid can be treated as incompressible, and we can neglect the effect of viscosity for the main body of the fluid. We shall assume the flow to be irrotational, so that a velocity potential $\phi$ exists, the gradient of which gives the velocity vector. In Cartesian coordinates $x, y$, and $z$, with $y$ measured in the direction of the vertical, the equation satisfied by $\phi$ is, as is well known,

$$
\phi_{x x}+\phi_{y y}+\phi_{z z}=0,
$$

where the subscripts indicate partial differentiation. The condition at any stationary solid boundary is

$$
\phi_{n}=0,
$$

* Received July 21, 1974. This work has been sponsored jointly by the National Science Foundation and the Office of Naval Research. 
where $n$ is measured in the direction of the normal to the boundary.

At the free surface there are a kinematic condition and a dynamic condition of constant pressure, which can be combined into ([1], p. 364)

$$
\phi_{\iota \iota}+g \phi_{\nu}=0 .
$$

We shall assume $\phi$ to have the time factor $\exp (-i \sigma t)$. Then $(2.3)$ can be written as

$$
\sigma^{2} \phi=g \phi_{\nu} .
$$

3. Comparison theorems for surface waves. The most obvious theorem presents itself when we compare one container with another one similar in shape but different in size. Let the first basin be $B$ and the second one be $B^{\prime}$, and let all linear dimensions of $B^{\prime}$ (including the depth of the liquid in it) be $m$ times that of $B$. If $\phi(x, y, z)$ is the solution for wave motion of any mode in $B$, then $\phi\left(m^{-1} x, m^{-1} y, m^{-1} z\right)$ is the solution for wave motion of the same mode in $B^{\prime}$, provided

$$
\sigma^{2}=m{\sigma^{\prime 2}}^{2}
$$

$\sigma$ being the frequency for $B$ and $\sigma^{\prime}$ the frequency for $B^{\prime}$. Thus we have

Theorem 1. If the size and liquid depth in a basin is multiplied by a factor $m$, the frequency of wave motion for any given mode is multiplied by a factor $(1 / m)^{1 / 2}$.

This result is fairly obvious, for on inspection of (2.1) to (2.4), we see that for any given geometry, $\sigma$ depends only on $g$ and the linear dimension $L$, so that $\sigma^{2} L / g$ must be constant. Indeed, we see also that $\sigma^{2}$ varies directly with $g$ for any given mode. But in conjunction with another, less obvious theorem it is very useful.

Moiseev [2] has given the following theorem:

Theorem 2. The frequency of water waves for any given mode in a basin of variable depth is always greater than that in another basin provided the free surface is exactly the same for both basins, the water-occupied domain in the former basin contains that in the latter basin, and no infinite velocity occurs in either basin.

We shall, for the sake of completeness, give the essential arguments of the proof. Let the first basin be denoted by $B^{\prime}$ and its water-occupied domain be denoted by $D^{\prime}$, and the second basin be denoted by $B$ and its water-occupied domain be denoted by $D$. It can be shown that an interior angle less than $\pi$ on the boundary of $B$ will in general give rise to a distributed singularity outside of $B$, but that that singularity distribution does not affect the conclusion. If there is an interior angle less than $\pi$ on the boundary of $B^{\prime}$, the result also remains, since the boundary of $B^{\prime}$ can be approached by a succession of smooth curves. Therefore we need only consider smooth boundaries for $B$ and $B^{\prime}$ here. Then there is no singularity of $\phi$ on the boundary of $B$ or of the continuation of $\phi$ infinitely close to it, since the velocity field along that boundary is finite and free from singularities, and we can take $B^{\prime}$ sufficiently close to $B$ so that $D^{\prime}-D$ is free from singularities. Since the solution $\phi^{\prime}$ for $B^{\prime}$ differs very little from the solution $\phi$ for $B$ and for the same mode, the projection of the gradient of $\phi^{\prime}$ on the gradient of $\phi$ must be everywhere positive in $D^{\prime}-D$, i.e.,

$$
P \equiv \phi_{x}{ }^{\prime} \phi_{x}+\phi_{\nu}{ }^{\prime} \phi_{\nu}+\phi_{z}{ }^{\prime} \phi_{z}>0,
$$

and, furthermore, over the free surface $S$ 


$$
Q \equiv \int_{S} \phi \phi^{\prime} d S>0 .
$$

Now $\phi^{\prime}$ must also satisfy (2.1), (2.2), and (2.4). We shall denote the three equations satisfied by $\phi^{\prime}$ by $\left(2.1^{\prime}\right),\left(2.2^{\prime}\right)$, and $\left(2.4^{\prime}\right)$, without explicitly displaying them. Multiplying $\left(2.1^{\prime}\right)$ by $\phi$, integrating over $D^{\prime}$, and using $\left(2.2^{\prime}\right)$ and $\left(2.4^{\prime}\right)$, we have

$$
\lambda^{\prime} Q=\int_{D^{\prime}} P d V,
$$

where $\lambda^{\prime}={\sigma^{\prime}}^{2} / g$. Similarly, multiplying (2.1) by $\phi^{\prime}$ and integrating over $D$, we have

$$
\lambda Q=\int_{D} P d V,
$$

where $\lambda=\sigma^{2} / g$. The difference between (3.4) and (3.5) is

$$
\left(\lambda^{\prime}-\lambda\right) Q=\int_{D^{\prime}-D} P d V .
$$

Since $P$ and $Q$ are positive, $\lambda^{\prime}>\lambda$. The process can be continued, and Theorem 2 follows.

A good many results follow from the two theorems above. For instance, we have

Theorem 3. If the horizontal dimensions of a basin is magnified (shrunk) by a constant factor, while the vertical dimension is unchanged, the frequency of any specified mode of water waves in it is reduced (increased).

We shall consider the case of horizontal magnification, since the case of horizontal shrinkage is entirely similar. Let the horizontally magnified basin be denoted by $B^{\prime}$ and the original basin by $B$. We introduce a third basin $B^{\prime \prime}$ obtained by shrinking $B^{\prime}$ in all directions uniformly, so that $B^{\prime \prime}$ is similar to $B^{\prime}$ but smaller, and in addition $B^{\prime \prime}$ and $B$ have exactly the same free surface. Then by Theorem 1 ,

$$
{\sigma^{\prime 2}}^{2}<{\sigma^{\prime \prime}}^{2},
$$

and since $D$ obviously contains $D^{\prime \prime}$ (water-occupied domain in basin $B^{\prime \prime}$ ), by Theorem 2

$$
{\sigma^{\prime \prime 2}}^{2}<\sigma^{2},
$$

and Theorem 3 follows.

Since trapezoidal channels occur in practice very often, we shall indicate how to obtain upper and lower bounds for the frequency of the first sloshing mode of water waves in such a channel. For an upper bound, we can use a rectangular channel with the same width at the free surface as the trapezoidal channel and a depth equal to the maximum depth of the trapezoidal channel. For a sharp lower bound, we select a streamline in a rectangular channel of width $b$ and depth $d$ which is tangent to the trapezoidal boundary (of the crosssection of the channel under consideration) at the free surface and at the horizontal bottom (see Figure 1). The $b$ and $d$ have to be calculated to achieve this. By Theorem 2, the frequency of waves in this curvilinear channel, which is exactly the same as that in the rectangular channel for the same mode, provides the lower bound needed. Since the curvilinear channel deviates from the trapezoidal channel mostly near its corners, where the velocity is small, the lower bound is likely to be near the actual frequency for waves in the trapezoidal channel. 


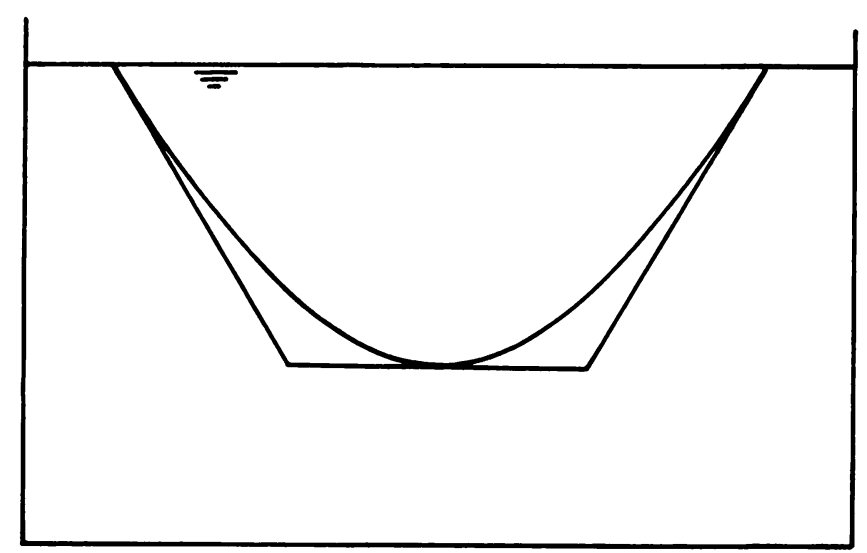

FIG. 1. The curve is a streamline in sloshing motion of the first mode in the rectangular channel. The frequency of that motion provides a sharp lower bound for the first sloshing mode in the trapezoidal channel. The curve is tangent to the trapezoid at three points: two at the level of the free surface, one at the middle of the horizontal bottom of the trapezoid.

4. Gravity waves in superposed layers of homogeneous fluids. For superposed homogeneous layers of different densities, within each layer (2.1) and (2.2) still are to be satisfied, and (2.4) is still the free-surface condition. At an interface the conditions are

$$
\sigma^{2}\left(\rho_{l} \phi_{l}-\rho_{u} \phi_{u}\right)=g\left(\rho_{l}-\rho_{u}\right) \phi_{\nu}, \quad\left(\phi_{l}\right)_{\nu}=\left(\phi_{u}\right)_{\nu}=\phi_{\nu} .
$$

where $l$ means "lower" and $u$ means "upper". One can easily verify that Theorem 1 is still true, and that Theorem 2 is still true if we require that the interfaces as well as the free surface must remain identical for the two basins under comparison. Under this requirement, we can compare two neighboring states as the vertical scale is slightly changed, and by continuation reach Theorem 3 . In this comparison, analytic continuations of $\phi_{l}$ or $\phi_{u}$ beyond an interface are needed if it is raised or lowered.

5. Differential system governing internal waves. Let the density in the absence of wave motion be $\rho_{0}(y)$, and the pressure be $p_{0}$. Then

$$
d p_{0} / d y=-g \rho_{0} .
$$

Let the velocity components in the directions of increasing $x, y$, and $z$ be denoted by $u, v$, and $w$, and let $p$ and $\rho$ be the perturbations in pressure and in density, respectively. The linearized equations of motion are, after (5.1) has been used,

$$
\begin{gathered}
\rho_{0} u_{t}=-p_{x}, \\
\rho_{0} v_{t}=-p_{\nu}-g \rho, \\
\rho_{0} w_{t}=-p_{z} .
\end{gathered}
$$

The linearized equation of incompressibility is

$$
\rho_{t}+v \rho_{0}^{\prime}=0,
$$

where the prime indicates differentiation with respect to $y$. The equation of continuity is then, since the fluid is incompressible, 


$$
u_{x}+v_{y}+w_{z}=0 .
$$

Again, the time factor exp $(-i \sigma t)$ will be assumed for all perturbation quantities. Then

$$
i \sigma \rho_{0} u=p_{x}, \quad i \sigma \rho_{0} w=p_{z},
$$

and combination of (5.3) and (5.5) gives

$$
v=-\frac{i \sigma p_{\nu}}{\sigma^{2} \rho_{0}+g \rho_{0}^{\prime}} .
$$

Substituting (5.7) and (5.8) into (5.6), we have

$$
p_{x x}+p_{z z}+\sigma^{2} \rho_{0} \frac{\partial}{\partial y} \frac{p_{y}}{\sigma^{2} \rho_{0}+g \rho_{0}{ }^{\prime}}=0 .
$$

If the direction numbers of the normal to a stationary rigid boundary are $(l, m, n)$, the boundary condition there is

$$
l u+m v+n w=0 .
$$

At a free surface the pressure is constant. But the pressure variation has two parts: the perturbation pressure $p$ and the variation of the mean pressure due to the freesurface displacement (denoted by $\eta$ ). Hence the condition on pressure at the free surface is

$$
p-g \rho_{0} \eta=0 .
$$

The kinematic condition at the free surface is

$$
v=\eta_{t} .
$$

Combination of (5.8), (5.11), and (5.12) gives

$$
p=\frac{g \rho_{0} p_{\nu}}{\sigma^{2} \rho_{0}+g \rho_{0}{ }^{\prime}} .
$$

It is well known, and it can be easily shown, that if $\rho_{0}{ }^{\prime}$ is never positive $\sigma^{2}$ is positive (i.e., $\sigma$ is real), and that, for truly internal waves,

$$
\sigma^{2}<-\max \left(g \rho_{0}{ }^{\prime} / \rho_{0}\right) .
$$

6. Comparison theorems for internal waves. For the case of a horizontal channel with depth varying with $z$ but not with $x$ (measured along the axis of the channel), the variation of $\sigma^{2}$ with $k^{2}$, with $k$ denoting the wave number in the $x$-direction, has been discussed by Yih [3], who found that for the same mode

$$
\frac{d \sigma^{2}}{d\left(k^{2}\right)}>0, \quad \frac{d c^{2}}{d\left(k^{2}\right)}<0,
$$

where $k c=\sigma$. We shall not repeat the discussion.

We shall now show

Theorem 4. $\sigma^{2}$ increases if $\rho_{0}$ is reduced by a constant or if both $\rho_{0}$ and $\rho_{0}{ }^{\prime}$ (henceforth assumed negative) are everywhere decreased, that is, if $\rho_{0}$ is everywhere decreased in such a way that $-\rho_{0}{ }^{\prime}$ is everywhere increased.

To prove this theorem let us compare two wave motions, one with 


$$
\rho_{0}=\rho_{1}, \quad p=p_{1}, \quad \sigma=\sigma_{1} .
$$

and the other with

$$
\rho_{0}=\rho_{2} \leq \rho_{1}, \quad p=p_{2}, \quad \sigma=\sigma_{2}, \quad \rho_{2}{ }^{\prime} \leq \rho_{1}{ }^{\prime}<0 .
$$

The two motions are near each other in the sense that $\rho_{2}$ is only slightly less than $\rho_{1}$ for all values of $y$, so that $\phi_{1}$ is nearly the same as $\phi_{2}$, and $\sigma_{1}$ nearly the same as $\sigma_{2}$.

We shall first treat the case in which no free surface or other density discontinuities exist. Consider the integral (with $V$ denoting the fluid volume, as before)

$$
\int_{V}\left[\left(\frac{1}{\rho_{1}} p_{2} p_{1 x}\right)_{x}+\left(\frac{1}{\rho_{1}} p_{2} p_{1 z}\right)_{z}+\sigma_{1}{ }^{2}\left(\frac{p_{2} p_{1 y}}{\sigma_{1}{ }^{2} \rho_{1}+g \rho_{1}{ }^{\prime}}\right)_{y}\right] d V,
$$

and another in which the subscripts 1 and 2 are exchanged. Since $p_{1}$ satisfies (5.9) with $\sigma$ replaced by $\sigma_{1}$ and $\rho_{0}$ by $\rho_{1}$, and $p_{2}$ satisfies (5.9) with $\sigma$ replaced by $\sigma_{2}$ and $\rho_{0}$ by $\rho_{2}$, (6.2) gives, upon use of the Gauss-Green theorem,

$$
\begin{aligned}
& i \sigma_{1} \int_{S} p_{2}\left(l u_{1}+m v_{1}+n w_{1}\right) d S \\
&=\int_{V}\left[\frac{1}{\rho_{1}}\left(p_{1 x} p_{2 x}+p_{1 z} p_{2 z}\right)+\frac{\sigma_{1}{ }^{2}}{\sigma_{1}{ }^{2} \rho_{1}+g \rho_{1}}{ }^{\prime} p_{1 \nu} p_{2 \nu}\right] d V,
\end{aligned}
$$

where $S$ is the surface of $V$. From the integral which is (6.2) with subscripts 1 and 2 exchanged, we obtain the result

$$
\begin{aligned}
i \sigma_{2} \int_{S} p_{1}\left(l u_{2}+m v_{2}+n w_{2}\right) & d S \\
= & \int_{V}\left[\frac{1}{\rho_{2}}\left(p_{1 x} p_{2 x}+p_{1 z} p_{2 z}\right)+\frac{\sigma_{2}{ }^{2}}{\sigma_{2}{ }^{2} \rho_{2}+g \rho_{2}} p_{1 \nu} p_{2 \nu}\right] d V .
\end{aligned}
$$

The left-hand sides of (6.3) and (6.4) are zero because of (5.10), the condition at the rigid boundary. The difference between (6.3) and (6.4) is

$$
\begin{aligned}
\int_{V}\left(\frac{1}{\rho_{2}}-\frac{1}{\rho_{1}}\right)\left(p_{1 x} p_{2 x}\right. & \left.+p_{1 z} p_{2 z}\right) d V \\
& +\int_{V} \frac{{\sigma_{1}}^{2}{\sigma_{2}}^{2}\left(\rho_{1}-\rho_{2}\right)+g\left({\sigma_{2}}^{2} \rho_{1}{ }^{\prime}-\sigma_{1}{ }^{2} \rho_{2}{ }^{\prime}\right)}{\left(\sigma_{2}{ }^{2} \rho_{2}+g \rho_{2}{ }^{\prime}\right)\left(\sigma_{1}{ }^{2} \rho_{1}+g \rho_{1}{ }^{\prime}\right)} p_{1 \nu} p_{2 \nu} d V=0 .
\end{aligned}
$$

Upon making $\rho_{2}$ close to $\rho_{1}, p_{1}$ is close to $p_{2}, \sigma_{1}$ is close to $\sigma_{2}$, etc. Then quantities like $p_{1 x} p_{2 x}$ and so on are positive everywhere except possibly near the stagnation points of the smaller basin, where their absolute values are small. Hence they are positive on the average. Then, since

$$
0<\rho_{2} \leq \rho_{1} \text { and } \rho_{2}{ }^{\prime} \leq \rho_{1}{ }^{\prime}<0,
$$

(6.5) shows that ${\sigma_{2}}^{2}$ must be greater than $\sigma_{1}{ }^{2}$.

If there is a free surface, the left-hand sides of (6.3) and (6.4) are, upon the use of the free-surface boundary condition (5.13), with $\rho_{0}$ identified with $\rho_{1}$ or $\rho_{2}, \sigma$ identified with $\sigma_{1}$ or $\sigma_{2}$,

$$
\int_{S^{\prime}} \frac{g \rho_{2} \sigma_{1}^{2} v_{1} v_{2}}{\sigma_{1} \sigma_{2}} d S^{\prime}, \quad \int_{S^{\prime}} \frac{g \rho_{1} \sigma_{2}^{2} v_{1} v_{2}}{\sigma_{1} \sigma_{2}} d S^{\prime}
$$


respectively, and the term

$$
\int_{S^{\prime}} \frac{g\left(\sigma_{1}{ }^{2} \rho_{2}-\sigma_{2}{ }^{2} \rho_{1}\right) v_{1} v_{2}}{\sigma_{1} \sigma_{2}} d S^{\prime}
$$

must be added to the right-hand side of (6.5), with $S^{\prime}$ denoting that part of $S$ that is the free surface. The conclusion that

$$
\sigma_{2}^{2}>{\sigma_{1}}^{2}
$$

therefore remains valid.

If there are internal density discontinuities terms like (6.8) but somewhat more complicated must be added to the right-hand side of (6.5), and the conclusion (6.9) remains valid.

Note that since the conclusion (6.9) is reached when (6.6) holds, by a comparison of neighboring states, it is automatically guaranteed that wave motions of the same mode are being compared. Furthermore, the restriction of neighboring states can be removed by continuation through neighboring states, so that the same mode is maintained while the two motions finally compared are no longer in neighboring states, that is, $\rho_{1}$ is no longer near $\rho_{2}$. (Mathematically speaking, $\rho_{1}$ being near $\rho_{2}$ means $\left(\rho_{1}-\rho_{2}\right) \rho_{1}^{-1} \ll 1$.)

Since for truly internal waves (5.14) holds, for small $\rho_{0}^{\prime}$ the vertical velocity at the free surface is very small, as can be seen from (5.13) and (5.8). Therefore when studying truly internal waves (i.e., with free-surface waves excluded) we can treat the free surface as rigid, and replace (5.13) by

$$
p_{\nu}=0 \text {. }
$$

We shall now assume $\rho_{0}$ to be continuous, and attempt to see what effect a change of shape or size of the fluid-occupied domain of a basin has on the frequency of internal waves. For this purpose we need to use the Boussinesq approximation, which amounts to ignoring the variation of $\rho_{0}$ except for the term $g \rho_{0}{ }^{\prime}$ in (5.8) and (5.9). Whether we consider an exponentially stratified fluid with

$$
\rho_{0}=C \exp (-\beta y)
$$

or a linearly stratified fluid with

$$
\rho_{0}=C(1-\beta y),
$$

after the Boussinesq approximation is made we can write (5.9) as

$$
p_{x x}+p_{z z}-\lambda^{2} p_{y y}=0
$$

where

$$
\lambda^{2}=\frac{\sigma^{2}}{g \beta-\sigma^{2}} .
$$

Let the boundary of the basin be given by

$$
F(x, y, z)=0 \text {. }
$$

For an open basin of the simplest shapes, $z$ is a single-valued function of $x$ and $y$. But it can be multi-valued. For a closed basin flowing full, it is at least double-valued. The condition at the boundary, (5.10), can now be written as 


$$
F_{x} p_{x}+F_{z} p_{z}-\lambda^{2} F_{\nu} p_{\nu}=0 .
$$

Since we treat a free surface as a rigid surface and therefore a part of (6.15), we can absorb (6.10) into (6.16).

Inspection of (6.13) and (6.16) gives

Theorem 5. With the actual $\rho_{0}$ given by (6.11) or (6.12) maintained unchanged (i.e., with $\beta y$ equal to $\beta^{\prime} y^{\prime}$ after a scale magnification or reduction), if any horizontal dimension of the basin is multiplied by $M$ and the vertical dimension by $N$, i.e., if $x, y$, and $z$ in $F(x, y, z)$ are replaced by

$$
x / M, \quad y / N, \quad z / M,
$$

then $\lambda^{2}$ is multiplied by $N^{2} / M^{2}$.

Hence if the widening exceeds the deepening, $\lambda^{2}$ (hence $\sigma^{2}$ ) is reduced, if the deepening exceeds the widening, $\sigma^{2}$ is increased, and if the magnification (or reduction) of scale is uniform in all directions, $M=N$, and $\sigma^{2}$ remains unchanged.

\section{REFERENCES}

[1] H. Lamb, Hydrodynamics, Cambridge Univ. Press, 6th Ed., 1932

[2] N. N. Moiseev, Adv. Appl. Mech. 8, 241 (1964)

[3] C. -S. Yih, Internal waves in channels of variable depth, presented to 9th Symposium of Naval Hydrodynamics, 1972, Paris 